\title{
The COVID-19 situation in Sudan. Plus, a possible life-saving intervention?
}

\author{
Hassan I. Osman ${ }^{* a}$, Meisa Elmamoun, MD ${ }^{* b}$, Ahmed A. Elgamel, PharmD *c \\ a hassan.io@live.co.uk \\ ${ }^{a, b}$ Napata College, Al-Riyadh 10-151, Khartoum, Sudan \\ c Forensic Science Institute, The National Ribat University, Khartoum - Sudan
}

\begin{abstract}
Purpose of review: To place forth a true understanding of the epidemiological characteristics of the novel coronavirus (COVID-19) in Sudan as well as propose a possible intervention that might decrease, if not completely deplete, the mortality rate. Recent findings: As of the $28^{\text {th }}$ of September 2020, as per the JHU's online world map covering the COVID-19 pandemic, the total number of confirmed cases worldwide was 33,469,217 with 1,003,791 deaths with the United States of America being the worst infected nation followed by Brazil and India (based on the total number of deaths); in the Republic of the Sudan, the Federal Ministry of Health (FMOH) reported by the $28^{\text {th }}$ of September 2020, a total of 13,640 cases, $836(\sim 6.1 \%)$ of these patients had died, $6764(\sim 49.6 \%)$ had recovered and the remaining 6040 $(\sim 44.3 \%)$ were classified as "active”. The republic has recently 'lifted' the previously imposed lockdown.
\end{abstract}


Published by IJRP.ORG. Selection and/or peer-review under responsibility of International Journal of Research Publications (IJRP.ORG)

Keywords: COVID-19; Coronavirus; Global Pandemic; Sudan; Africa; Immunology;

\section{Main text}

\subsection{Introduction:}

The COVID-19 (coronavirus disease) pandemic, which was identified as the cause of the respiratory disease in Wuhan, China in December 2019, has resulted in the crashing of global economies, the spread of fear amongst the entirety of the planet and, of course, the overwhelming of healthcare systems around the globe. Here, we plan on providing an understanding of the history associated with the pandemic, what its signs and symptoms are, and, as is indicated by the title, the epidemiology of COVID- 19 in the nation of Sudan (The Republic of The Sudan). We will also be discussing the proposed treatments as well as proposing our own, in addition, we will be making recommendations on how to prevent the re-occurrence of a global pandemic.

\subsection{History:}

The coronavirus is a member of a family of viruses well known to cause respiratory illnesses such as breathing difficulty, pneumonia and lung infection ${ }^{[1]}$. It has been known for a considerable amount of time now that such infections are zoonotic in nature (i.e.: occur in animals and then transmit to humans), but are rather rare as far as their occurrence in humans is concerned. In December of 2019, a report by Zhu and colleagues which were published in the New England Journal of Medicine (NEJM), was made public, the report illustrated a peculiar and unfamiliar pneumonia of unknown cause; although the pneumonia was of unknown cause, it was traced to a local Huanan South China Seafood Market in Wuhan, China [2]. The WHO coined the term "2019 novel coronavirus" to refer to the unfamiliar virus at first, after which it announced the official nomenclature of the virus being "coronavirus disease (COVID-19)" [1,3].

With the notion of this being a possible outbreak, the Chinese CDC prepared and transmitted a RRT to assess the situation and aid health officials in Wuhan with the conduction of the appropriate epidemiological investigations ${ }^{[1]}$. Although, no particular association with any animal species was recognized, the WHO confirmed the aforementioned association between the Huanan South China Seafood Marketplace and the virus ${ }^{[1,4]}$. On the $10^{\text {th }}$ of January 2020, Zhang YZ and his research team published the first genome of the COVID19 virus ${ }^{[1,5]}$. Seeing as how this outbreak corresponded with the Chinese New Year (a period characterized by high extroverted activities by the Chinese), the virus spread rapidly throughout China ${ }^{[1]}$. By the $30^{\text {th }}$ of January 2020, the WHO declared COVID-19 a "Public Health Emergency of International Concern" [6]. The virus is 
also referred to as "severe acute respiratory syndrome coronavirus 2 (SARS-CoV-2)" ${ }^{[1]}$.

As will later on be described, this is not the first "coronavirus" epidemic; in the previous 2 decades SARS and MERS were also coronavirus epidemics ${ }^{[1]}$.

\subsection{Virology:}

As aforementioned, coronaviruses are zoonotic in nature. They are enveloped single-stranded RNA viruses and cause result in a plethora of symptoms ranging from the common cold to severe respiratory, hepatic, enteric and neurological in their manifestations ${ }^{[1]}$. In total, there are, in addition to the COVID-19 virus, 6 coronaviruses that we know of; those being:

1) $\mathrm{HCoV}-229 \mathrm{E}$

2) $\mathrm{HCoV}-\mathrm{OC} 43$

3) SARS-CoV

4) HCoVNL63

5) HCoV-HKU1

6) MERS-CoV

For obvious reasons, we will not be discussing each of the above viruses in detail.

The aforementioned Chinese CDC research team collected a total of 585 environmental samples from the Hana Seafood Market in Wuhan on the $1^{\text {st }}$ and $12^{\text {th }}$ of January 2020. Following appropriate investigations, they found a total of 33 samples containing the virus and concluded that it originated from the animals being sold in the market ${ }^{[1]}$. Following this, the researchers collected lung fluid, throat and blood samples form 15 patients with the intent of conducting needed laboratory investigations; the investigations confirmed a new form of coronavirus species which was similar to a collection of $\beta$ coronaviruses genera found in bats, which is situated in a group of SARS/SARS-like COV ${ }^{[1]}$. Adhikari and colleagues published a paper which beautifully illustrated the following findings, as a result we will be copying their paper ${ }^{[1]}$ :

"To conduct next-generation sequencing from bronchoalveolar lavage fluid and cultured isolates, researchers enrolled nine inpatients in Wuhan with viral pneumonia and negative in common respiratory pathogens. The results of this next-generation sequencing indicated that SARS-CoV-2 was more distant from SARS-CoV (with about 79\% sequence identity) and MERS-CoV (with about 50\% sequence identity) than from two bat-derived SARS-like coronaviruses - bat-SL-CoVZC45 (with 87.9\% sequence identity) and bat-SL-CoVZXC21 (with 87.2\% sequence identity). Studies also reported that COVID-19 S-protein supported strong interaction with human ACE2 molecules despite the dissimilarity of its sequence with that of SARS-CoV." 
1.4: How is the coronavirus transmitted?

As aforementioned, the virus is zoonotic in nature, which indicates its presence in non-human animals. A number of animals can serve as hosts for coronaviruses these include camels, cats, bats and cattle ${ }^{[1,7]}$. In a general sense, animal coronaviruses do not spread amongst humans ${ }^{[8]}$, with the existence of certain exceptions such as the SARS and MERS viruses which are primarily spread through close contact with the infected via droplets of the respiratory system (e.g. cough) ${ }^{[1]}$. When the virus first became manifest and was traced back to the market; the consensus was that the infections were a result of animal-to-human pathway of infection [1]. However, later cases involving HCWs were reported in which the patients were not exposed to the market (some haven't even visited Wuhan), which gave rise to the idea that the virus is transmissible via humans without the need for exposure to animals (human-to-human transmission) ${ }^{[1]}$.

In addition to the virus being transmitted via the respiratory system, a study illustrated the possibility of the GIT being a possible route of transmission ${ }^{[9]}$. The researchers of the above study justified their finding that ACE2 was highly expressed in absorptive electrolytes from the ileum and colon ${ }^{[1,9]}$.

1.5: How does the virus clinically manifest itself?

As is the case with all disorders, you can never ascertain the existence of a disease only via the clinical manifestations. With that being said, it is important that we are aware of how it might clinically present.

Given how New York City, NY, USA has in a rather rapid manner, risen as a COVID-19 epicenter, we will be discussing the findings associated with the outbreak in one of the world's largest cities.

On the $17^{\text {th }}$ of April 2020, physicians at Weill Cornell Medicine (Medical school in NYC) published a case series in which the clinical characteristics of COVID-19 in NYC patients. Their findings were as follows:

1) The median age of patients was 62.2 years

2) $60.6 \%$ of their patients were male

3) $35.8 \%$ were obese (obesity was defined as a BMI of over 30 )

4) $79.4 \%$ presented with cough

5) $77.1 \%$ presented with fever 
6) $56.5 \%$ presented with dyspnea

7) $23.8 \%$ presented with myalgias

8) $23.7 \%$ presented with diarrhea

9) $19.1 \%$ presented with nausea and vomiting

10) $90 \%$ had lymphopenia

11) $27 \%$ had thrombocytopenia

12) $33.1 \%$ of their patients developed respiratory failure requiring invasive mechanical ventilation,

$33.1 \%$ of these patients were extubated.

13) $10.2 \%$ of all the patients were deceased

14) $66.2 \%$ of all the patients were discharged from hospital

15) Data was incomplete for the remainder $23.7 \%$

16) Obese males were more likely to require mechanical ventilation

For reasons that are, yet, unclear, the case series in New York illustrated more symptoms associated with the GIT. With that exception, the above report from New York ${ }^{[10]}$ is remarkably similar to a report out of China [11].

A rather frightening case series by Bangalore and colleagues ${ }^{[12]}$ illustrated the presence of ST-segment elevation on ECG/EKG investigation which is indicative of acute MI. Half of their patients underwent coronary angiography, which presents an increased overload on healthcare systems.

A case report out of France ${ }^{[13]}$ also illustrated the presence of immune thrombocytopenic purpura in a female patient with autoimmune hypothyroidism.

\section{6: COVID-19 and pregnancy:}

Although COVID-19 has taken the world by storm, not much data is available discussing its association with pregnancy. Thankfully, Chen $\mathrm{L}$ and colleagues ${ }^{[14]}$ decided to address this issue and took it upon themselves to study the association between COVID-19 and pregnancy by studying a population of 118 pregnant women (note that $29 \%$ of these women had "suggestive" chest CT findings). Of the population that was being studied, $0.24 \%$ were pregnant women. $52 \%$ of these women were nulliparous with a median age of 31 years. $64 \%$ of them were infected with the virus in the $3^{\text {rd }}$ trimester of their pregnancies. $79 \%$ of the women who underwent CT had "infiltrates in both lungs" [14].

The study reported that $8 \%$ of the patients "had severe disease" ${ }^{[14]} .0$ deaths were reported.

Of those who delivered during the study (58\%), 93\% underwent caesarian section, $61 \%$ of which were performed as a result of concern of the possible effects of COVID-19 on pregnancy ${ }^{[14]}$. The study also reported: 
"Among the study population, there were 3 spontaneous abortions, 2 ectopic pregnancies, and 4 induced abortions (all owing to patients' concerns about Covid-19)." [14]

"Testing for SARS-CoV-2 was performed on neonatal throat swabs of 8 newborns and breast milk samples of 3 mothers. No positive results were reported." [14]

\section{7: Situation in Sudan}

Sudan (The Republic of The Sudan) was one of the later nations to become introduced to COVID-19. As of the $14^{\text {th }}$ of March 2020, the Sudanese government announced the bringing of the educational process to a halt, after which a curfew was announced, and finally a total 3-month long shutdown of the nation was announced by the government. By the $25^{\text {th }}$ of April 2020, a total of 213 cases were reported by the Sudanese FMOH, 17 ( 8\%) of these patients had died, $19(\sim 9 \%)$ had recovered and $177(\sim 83 \%)$ were classified as "active". In addition to that, the cases were scattered over 7 of the nation's states, with the vast majority $(\sim 94 \%)$ of those cases were situated within the state of Khartoum, within which the capital city of Khartoum is situated. Although Khartoum is central in its geography, a case was reported in the state of Red Sea which is situated in the Eastern division of the nation indicating, in our estimation, that the virus has spread throughout the nation without exception. By the $15^{\text {th }}$ of June 2020, the number of cases totaled 8,020; as aforementioned in the abstract; with 487 deaths and a total of 2,966 recoveries. It is highly important that we note that, although the nation is in a state of lockdown (shutdown), the number of cases keeps rising which might be indicative of 1) the virus being highly infectious, and 2) the possibility of needing to consider extending the shutdown. By the $23^{\text {rd }}$ of August 2020, the total number of cases had risen to 12,974, the illness had claimed 819 lives and a total of 6,557 individuals had recovered. As had been the case, the vast majority of cases were in the state of Khartoum $(9,299)$, followed by the state of Gezira $(1,115)$. The state with the least number of cases was that of Middle Darfur which, as of the $23^{\text {rd }}$ of August 2020, had a total of 6 cases and 0 deaths. The numbers might seem to indicate that the Republic has controlled the virus quite well.

Currently, as of the $28^{\text {th }}$ of September 2020, the situation is as follows: the total number of cases is at 13,640 ; the number of deaths is at 836 and the number of recoveries is at 6,764. According to the same report by the Sudanese FMOH, out of a total of 901 tested samples, only 27 tested as positive, a cumulative percentage of $3 \%$.

Unfortunately, we hypothesize that is not the case. Our hypothesis is justified by an apparent lack of testing by the federal government. We suspect the numbers to be deceiving of the true extent to which the illness has infiltrated the Republic of the Sudan. 
1.8: A possible life-saving treatment?

The virus enters the body's cells via the ACE2 (angiotensin-converting enzyme-related carboxypeptidase) inhibitor ${ }^{[15]}$. This receptor is highly expressed in cardiopulmonary tissues ${ }^{[15]}$ (which may explain the STsegment elevation reported by Bangalore and colleagues ${ }^{[12]}$ (discussed above)) as well as some blood cells (e.g: macrophages and monocytes) ${ }^{[15]}$ (which may explain our theory of COVID-19 being associated with strokes).

In other coronaviruses (namely MERS-CoV), IL-6 are hallmarks of severe infection ${ }^{[15]}$.

It has been indicated that Cytokine Release Syndrome (CRS) is a rather common phenomenon in COVID-19 patients, it is also important that we note the association between IL-6 elevations and ARDS and respiratory failure ${ }^{[15,16,17]}$ (a common cause of death amongst COVID-19 patients). In addition, the elevation of CRP, which is expressed via IL-6 is also an indicator of "severe betacoronovirus infection" [15]. 2 alarming findings out of a retrospective study by Ruan and colleagues ${ }^{[17]}$ reported 2 crucial findings as it relates to our understanding of COVID-19, those were that:

elevated serum ferritin, and

elevated serum IL-6

were correlated with mortality amongst COVID-19 patients.

Moore and June ${ }^{[15]}$ make a great case for the use of Tocilizumab (IL-6R antagonist) in patients suffering from CRS via narrating the story of Emily Whitehead which goes as follows:

"Emily Whitehead, the first patient to receive CD19-targeted CAR T cells to treat pediatric B cell acute lymphoblastic leu-kemia in 2012, developed severe CRS and sHLH, leading to ARDS with multiorgan failure and hypotension that was refractory to standard treatment with steroids. Because of greatly elevated serum IL6 in this patient, she was treated empirically with tocilizumab, an IL-6R antagonist ap-proved at the time to treat rheumatic conditions such as juvenile idiopathic arthritis. She received a single dose of tocilizumab on day 7 after CAR T cell administration, with rapid resolution of fever within hours followed by weaning from vasopressors (which treat hypotension) and from ventilator support as ARDS resolved." 
Tocilizumab is an FDA approved drug for use in treating CAR T cell-induced CRS. We, hereby, urge the immediate implementation of a research project testing the effectiveness of Tocilizumab in reducing the CFR of COVID-19.

On the $16^{\text {th }}$ of April 2020, The Society for Immunotherapy of Cancer published ${ }^{[18]}$ a statement urging for the implementation of

"IL-6-taregting” therapies (such as Tocilizumab) for COVID-19. The following is a quote from said statement:

"Emerging evidence suggests that high levels of C reactive protein (CRP) and IL-6 are observed in patients infected with COVID-19. Anecdotal experience on the use of tocilizumab at doses comparable to those used for the management of CRS from investigators in Italy and from China has reported rapid improvement in both intubated and non-intubated patients. In these reports, expeditious administration of anti-IL-6R therapy for patients in acute respiratory distress has been critical. A recent study protocol to evaluate the efficacy of tocilizumab in COVID- 19-induced pneumonitis accrued over 300 patients worldwide in less than 24 hours. Additionally, Genentech will

also provide 10000 vials of tocilizumab to the US Strategic National Stockpile.11 Tocilizumab was also approved in China in March 2020, for the treatment of patients with COVID-19 with serious lung damage and elevated IL-6. Sponsors, investigators and regulators have moved with unprecedented speed and collaboration to initiate protocols to formally study the safety and efficacy of antiviral agents and vaccines, as well as various anti-IL-6 antibodies in patients with COVID-19. In the USA, a trial of sarilumab in the COVID-19 setting is ongoing."

Another interesting quote from the statement ${ }^{[18]}$ :

"Now, data from the frontlines of the pandemic indicates that the agent may offer lifesaving benefit for COVID19 patients with respiratory distress."

1.9: Possible side effects:

As is always the case with an intervention of the kind proposed here, there exists a possibility of adverse (side) effects manifesting themselves in a rather unexpected manner. In an attempt to avoid side effects taking practitioners by surprise, we judged that it would only be appropriate that we list the possible effects here. These notion was alluded to by Sheppard and colleagues in their 2017 paper titled 'Tocilizumab (Actemra)' ${ }^{[22]}$. In that paper, the following is mentioned: 
"Particular care should be exercised with concomitant medication that has a narrow therapeutic index such as theophylline, warfarin, phenprocoumon, phenytoin or ciclosporin.5 It should also be noted that due to the long half-life of TCZ monitoring of this interaction may be necessary for 1 to 2 months after the TCZ is discontinued.5

The elimination of TCZ has been shown to be relatively slow and concentration dependent. After saturation of IL6 receptors, clearance medicated by the mononuclear phagocyte system becomes linear. Increases in the dose have been shown to lead to prolongation of the half-life suggesting that the elimination of TCZ is capacity limited.40" [22]

The article goes on to state the following:

"Adverse effects (AEs) have been documented for TCZ monotherapy or in combination with other DMARDs.5 Safety data are not available regarding combination with other biologic therapy. 5 The most common AEs reported were infections.5 Skin infections were more common in TCZ group in the

AMBITION trial.43 Five serious infections were reported in TOWARD trial: staphylococcus cellulitis, acute pyelonephritis and sepsis in the TCZ group and 2 cases of pneumonia in the control group. 45 Infection rates from phase 3 trials (OPTION, TOWARD, LITHE and RADIATE) where patients received both DMARDs and TCZ or placebo, showed no evidence of increased risk with continued TCZ therapy.41,42,44,45 The TENDER

trial reports 4 serious $\mathrm{AE}$ in the TCZ arm of the study in 3 patients and this included angioedema and urticaria (one), varicella (one), and bacterial arthritis. 48

Gastrointestinal disorders included nausea; abdominal pain, mouth ulceration and gastritis were the second most common AEs. Perforations are also reported mostly in patients who have been diagnosed with diverticular disease before. One patient reported to have died in AMBITION trial in the TCZ group because of upper gastrointestinal hemorrhage and perforation

Other events occurring more frequently in the TCZ group included headache and hypertension.45" [22] 
In addition, the following is also stated:

It is critically important that all healthcare professionals remain responsible for ensuring appropriate pharmacovigilance

of adverse drug reactions (ADRs). The appropriate national regulators should always be informed of ADRs at the earliest

available opportunity and healthcare professionals should be responsible for including as much detail as possible in their reports to aid in their overall assessment and classification. Because of the likely structural batch to batch variation with biologic medicinal products it is critically important to include

details of the brand name and batch number of the medication. The results of one Dutch study have suggested despite this being an EU policy in reality this information is rarely recorded.56 To facilitate this, appropriate systems must be in place to record both the brand names and batch numbers of all biologics administered to all patients." [22]

1.10: A summarization of our proposal:

The COVID-19 global pandemic has taken the world by storm. So far, it has claimed numerous lives (mentioned above) and has resulted in a plethora of secondary medical, societal and economic disparities. It has also, in a sense, exposed a number of issues that exist with our perception of how healthcare systems should operate as well as how detrimental it is to the wellbeing of our species. The methodology by which COVID-19 claims lives is not singular in nature. That is understandable, given the uniqueness of humans and how each and every one of us is at least slightly (sometimes significantly) different from one another in our reaction to drugs, alcohol, medications and foods. With that being said however, there are medications that have been found safe enough to administer on a massive scale and that is something that can be applied here.

So, how does COVID-19 claim human lives? Xi, Ma, Tang and Liu ${ }^{[19]}$ published a review article back in May of 2020 in which they illustrated the following:

"The damage mechanism of SARS-CoV-2 is still unclear. It is possible that protein S binds to angiotensinconverting enzyme 2 receptors and invades alveolar epithelial cells, causing direct toxic effects and an excessive 
immune response. This stimulates a systemic inflammatory response, thus forming a cytokine

storm, which leads to lung tissue injury." [19]

Cytokine storms are the result of what is known as CRS. As illustrated in the 2018 article by ShimabukuroVornhagen and colleagues ${ }^{[20]}$ :

"Depending on a number of characteristics of the host, the tumor, and the therapeutic agent the administration of $\mathrm{T}$ cell-engaging therapies can set off an inflammatory circuit that overwhelms counter-regulatory homeostatic mechanisms and results in a cytokine storm that can have detrimental effects on the patient." ${ }^{[20]}$

As aforementioned, our proposed treatment targets IL-6. Our justification for doing so is because it has been well established that IL-6 plays a major role in CRS and all that it entails ${ }^{[20]}$. Not only that, it also seems as if the cardiac symptoms we mentioned may be associated with elevations of IL-6 ${ }^{[20]}$.

Tocilizumab seems to be of immense benefit to those experiencing cytokine storms. This notion is superbly illustrated by Tanaka, Narazaki and Kishimoto in their very-well written 2016 article ${ }^{[21]}$, in that article, they write the following:

"However, dramatic beneficial effects of one injection of tocilizumab for patients with cytokine release syndrome (CRS), induced by T-cell engaged therapy, strongly suggest that IL- 6 blockade may constitute an innovative therapeutic approach for an acute, severe systemic inflammatory response known as a "cytokine storm'. This review focuses on the pathological role of IL-6 in a cytokine storm and the therapeutic effect of IL-6 blockade strategy on such an inflammatory response." [21]

By now, we have established the following:

1) Cytokine Storms are a highly plausible cause of death amongst severely-ill COVID-19 patients,

2) Cytokine Storms are the results of CRS

3) IL-6 plays a major role in CRS and, ergo, cytokine storms, and

4) Suppressing IL-6 may be life-saving in such scenarios as it prevents the unfortunate life-threatening cascade that takes place.

Does this mean that we are certain of IL-6 targeting therapies, such as Tocilizumab, will manifest themselves as the solution to the pandemic that has taken the planet by storm? No, we are not. However, we are certain that moving forward with this notion will definitely help improve our understanding of how this pandemic can be brought to a halt. 
1.11: Recent findings:

Back in early August of 2020, Mock and colleagues published the very smartly titled 'COVID-19: Clean up on IL-6' ${ }^{[23]}$. In that paper, the authors discussed how the current approach to the COVID-19 global pandemic, particularly the one associated with IL-6-targetting therapies, such as tocilizumab was with 'ill-defined criteria for use' ${ }^{[23]}$. As per the paper, 'As such, it remains unknown whether elevated IL-6 in viral infections represents a therapeutic target or part of a functioning adaptive immune response.'. The primary aim of the paper is to bring into attention the fact that our understanding of the role of IL-6 in inflammation. With that being said, it is important that we note that one of our proposed inclusionary criteria is an elevated serum IL-6 level. Anyhow, we propose the advancement of our understanding of IL-6 and how it functions. However, we want to bring to attention the fact that this is an emergency scenario.

1.12: Recommendations:

We hereby recommend the following:

1) For our proposal to be assessed and for our study to be permitted to take place as soon as possible.

2) Increased funding of scientific research targeting COVID-19 in Sudan and all around the globe.

3) The immediate implementation of research in the effectiveness of Tocilizumab on severely-ill COVID19 patients.

4) Extension of the stay-at-home order implemented within the entirety of the Republic.

5) The implementation of stricter so-called "social-distancing" strategies.

6) The implementation of follow-up programs targeting those who arrived so-called "COVID-19 hotspots" as well as all those who came into contact with them.

7) The implementation of a Federal Aid program targeting those who were adversely effected by the pandemic so as to urge them not to go to work.

8) The introduction of increased amounts of PPE into the healthcare system.

9) The implementation of programs to prevent the re-occurrence of epidemics which have previously become manifest throughout the nation.

10) As previously promised, we will be recommending the following so that we may potentially prevent (or at least severely decrease the effect of) future pandemics. The following are recommendations to do so.

11) The normalization of hygienic measure (washing hand when you enter your home, wearing masks and staying home whenever you feel symptoms of respiratory illness, etc.)

12) Increased funding for community medicine departments worldwide

13) Increased research on the best educational strategies

14) Creating a global taskforce that functions in the prompt detection and possible prevention of future outbreaks of this nature (to be run by institutions such as the WHO) as well as funding said taskforce properly.

15) Increasing access to evidence-based medical papers (funding journals so that all research may be openaccess and free of publication fees for authors) 
16) Encouraging medical students and junior practitioners to further contribute to the world of research (maybe even making it mandatory)

17) The establishment of a yearly conference (preferably virtual and free of charge) in which all medical personnel may participate and allocating further resources to nations (e.g. Sudan) which have poor rates of contribution to medical research. 
2. Abbreviations:

3. $\mathrm{MD}=$ Medical Doctor/Doctor of Medicine

4. JHU = Johns Hopkins University

5. FMOH $=$ Federal Ministry of Health

6. CFR = Case Fatality Rate

7. NEJM = New England Journal of Medicine

8. WHO = World Health Organization

9. $\mathrm{CDC}=$ Center for Disease Control and Prevention

10. RRT $=$ Rapid Response Team

11. SARS = Severe Acute Respiratory Syndrome

12. MERS $=$ Middle Eastern Respiratory Syndrome

13. $\mathrm{HCW}=$ Health Care Worker

14. GIT = Gastrointestinal Tract

15. $\mathrm{NY}=$ New York

16. USA = United States of America

17. BMI $=$ Body Mass Index

18. ECG/EKG $=$ Electrocardiogram

19. $\mathrm{MI}=$ Myocardial Infarction

20. $\mathbf{C T}=$ Computed Tomography

21. IL-6 = Interleukin-6

22. CRS = Cytokine Release Syndrome

23. ARDS = Acute Respiratory Distress Syndrome

24. $\mathbf{C R P}=\mathrm{C}$-reactive Protein 


\section{CRS = Cytokine Release Syndrome}

\section{References:}

1) Sasmita Poudel Adhikari, et. al. Epidemiology, causes, clinical manifestation and diagnosis, prevention and control of coronavirus disease (COVID-19) during the early outbreak period: a scoping review. Infectious Diseases of Poverty (2020) 9:29 https://doi.org/10.1186/s40249-02000646-x

2) Zhu N, Zhang D, Wang W, Li X, Yang B, Song J, et al. A novel coronavirus from patients with pneumonia in China, 2019. N Engl J Med. 2020. https://doi.org/10.1056/NEJMoa2001017.

3) WHO. Novel Coronavirus-China. 2020. https:// who.int/csr/don/12- january-2020-novel-coronavirus-china/en. Accessed 4 April 2020.

4) WHO. Novel Coronavirus-Japan (ex-China). 2020. http:// who.int/csr/don/17-january-2020-novel-coronavirus-japan-ex-china/en/. Accessed 5 April 2020

5) Virological.org. Novel 2019 Coronavirus Genome 2020. http://virological.org/t/novel-2019coronavirus-genome/319. Accessed 5 April 2020.

6) WHO. Novel Coronavirus-China. 2020. http://who.int/emergencies/diseases/novel-coronavirus2019/events-as-they-happen. Accessed 23 April 2020

7) National Health Commission of People's Republic of China. Prevent guideline of 2019-nCoV. 2020. http://www.nhc.gov.cn/xcs/yqfkdt/202001/bc661e49b5bc487dba182f5c49ac445b.shtml. Accessed 3 Feb 2020

8) CDC. 2019 Novel coronavirus, Wuhan, China. 2020. https://www.cdc.gov/coronavirus/2019nCoV/summary.html. Accessed 3 Feb 2020

9) Zhang H, Kang Z, Gong H, Xu D, Wang J, Li Z, et al. The digestive system is a potential route of 2019-nCov infection: a bioinformatics analysis based on single-cell transcriptomes. bioRxiv. 2020; doi: https://doi.org/10.1101/2020.01.30.927806.

10) Goyal P, et. al. Clinical Characteristics of Covid-19 in New York City. N Engl J Med. 2020. DOI: 10.1056/NEJMc2010419

11) Guan W, Ni Z, Hu Y, et al. Clinical characteristics of coronavirus disease 2019 in China. N Engl J Med. 2020. DOI: 10.1056/ NEJMoa2002032.

12) Bangalore S, et. al. ST-Segment Elevation in Patients with Covid-19 - A Case Series. N Engl J Med. 2020. DOI: 10.1056/NEJMc2009020

13) Zulfiqar AA, et al. Immune Thrombocytopenic Purpura in a Patient with Covid-19. N Engl J Med. 2020. DOI: 10.1056/NEJMc2010472

14) Chen L, et. al. Clinical Characteristics of Pregnant Women with Covid-19 in Wuhan, China. N Engl J Med. 2020. DOI: 10.1056/NEJMc2009226

15) J. B. Moore, C. H. June, Science 10.1126/science.abb8925 (2020).

16) G. Chen, D. Wu, W. Guo, Y. Cao, D. Huang, H. Wang, T. Wang, X. Zhang, H. Chen, H. Yu, X. Zhang, M. Zhang, S. Wu, J. Song, T. Chen, M. Han, S. Li, X. Luo, J. Zhao, Q. Ning, Clinical and immunological features of severe and moderate coronavirus disease 2019. J. Clin. Invest. 137244 (2020). doi:10.1172/JCI137244 Medline

17) Q. Ruan, K. Yang, W. Wang, L. Jiang, J. Song, Clinical predictors of mortality due to COVID-19 based on an analysis of data of 150 patients from Wuhan, China. Intensive Care Med. 10.1007/s00134-020-05991-x (2020). doi:10.1007/s00134-020-05991-x Medline 
18) Ascierto PA, Fox B, Urba W, et al. Insights from immuno-oncology: the Society for Immunotherapy of Cancer Statement on access to IL-6-targeting therapies for COVID-19. Journal for ImmunoTherapy of Cancer 2020;8:e000878. doi:10.1136/ jitc-2020-000878

19) Xie P, Ma W, Tang H and Liu D (2020) Severe COVID-19: A Review of Recent Progress With a Look Toward the Future. Front. Public Health 8:189. doi: 10.3389/fpubh.2020.00189

20) Shimabukuro-Vornhagen et al., Cytokine Release Syndrome, Journal for ImmunoTherapy of Cancer (2018) 6:56 https://doi.org/10.1186/s40425-018-0343-9

21) Tanaka T., Narazaki M., Kishimoto T., Immunotherapeutic implications of IL-6 blockade for cytokine storm, Immunotherapy (2016) 8(8), 959-970

22) Sheppard M, et. al. Tocilizumab (Actemra). HUMAN VACCINES \& IMMUNOTHERAPEUTICS 2017, VOL. 13, NO. 9, 1972-1988 https://doi.org/10.1080/21645515.2017.1316909

23) Mock JR, et. al. COVID-19: Clean up on IL-6. AJRCMB. 2020. 10.1165/rcmb.2020-0277LE 